\title{
The aging clock: circadian rhythms and later life
}

\author{
Suzanne Hood ${ }^{1}$ and Shimon Amir ${ }^{2}$ \\ 'Department of Psychology, Bishop's University, Sherbrooke, Quebec, Canada. ²Department of Psychology, Concordia University, Montreal, Quebec, Canada.
}

\begin{abstract}
Circadian rhythms play an influential role in nearly all aspects of physiology and behavior in the vast majority of species on Earth. The biological clockwork that regulates these rhythms is dynamic over the lifespan: rhythmic activities such as sleep/ wake patterns change markedly as we age, and in many cases they become increasingly fragmented. Given that prolonged disruptions of normal rhythms are highly detrimental to health, deeper knowledge of how our biological clocks change with age may create valuable opportunities to improve health and longevity for an aging global population. In this Review, we synthesize key findings from the study of circadian rhythms in later life, identify patterns of change documented to date, and review potential physiological mechanisms that may underlie these changes.
\end{abstract}

\section{Introduction}

Worldwide, the human population is graying rapidly: between 2010 and 2050, the proportion of adults over 65 years of age will double from $8 \%$ to $16 \%$ of the global population (1). Aging affects all aspects of our physiology and behaviors, including the circadian clock. Although the processes underlying aging are not well understood, increasing evidence suggests that the circadian system influences aging and longevity in important ways $(2,3)$.

Circadian rhythms play a vital role in health, and prolonged disruptions to the clock are associated with negative health consequences. With increasing age, the circadian system undergoes significant changes that affect rhythms of behaviors, temperature regulation, and hormone release, to name only a few examples. Although many of these changes may be an inevitable part of development, others may perhaps represent the operation of pathological processes that simply correlate with age. Improved understanding of the distinction between these concepts may create valuable opportunities to intervene and improve quality of life. This is particularly important in light of mounting evidence that disrupted circadian rhythms are an early warning sign of developing neurodegenerative diseases, such as Alzheimer's, Parkinson's, and Huntington's (3-5), and that the circadian clock is heavily implicated in the etiology of metabolic diseases, chronic inflammation, and cancers (6-9).

In this Review, we survey some of the key age-associated changes in circadian rhythms of behavior, physiology, and molecular processes. We then consider some of the central hypotheses of what underlies these changes, and summarize evidence for putative mechanisms of action.

\section{Cellular basis of circadian rhythms}

Circadian rhythms provide an adaptive mechanism for organisms to coordinate cellular processes, physiological functions, and behaviors with the predictable 24-hour cycle of light and dark on Earth (10). In mammals, the suprachiasmatic nucleus (SCN) is the

Conflict of interest: The authors have declared that no conflict of interest exists. Reference information: J Clin Invest. 2017;127(2):437-446.

https://doi.org/10.1172/JCI90328. master circadian clock. Inputs to the SCN from the retinohypothalamic tract synchronize the endogenous clockwork to light cues from the external environment (11). In turn, the SCN communicates time-of-day information by synaptic and diffusible signals to clocks in various brain regions and peripheral organs including the heart, lungs, liver, and endocrine glands (12). Thus, the SCN serves to synchronize the timing of rhythmic activities throughout the body to the light/dark cycle (13).

At a cellular level, the circadian clock comprises a core group of genes that regulate their own transcription and translation over 24 hours via a series of interacting negative-feedback loops (Figure 1 , and reviewed in refs. 13,14). In addition to regulating their own levels of expression in a 24-hour cycle, "clock" genes serve as transcription factors for a wide variety of other genes implicated in a host of functions (15).

\section{Age-associated changes in circadian rhythms}

Observations of many species have revealed a variety of developmental changes in circadian rhythms of overt behaviors and physiology (see Figure 2 for an illustration of changes in human rhythms). In reviewing this literature, it is worthwhile to note some aspects of research design that may influence the conclusions that can be drawn. For example, the vast majority of research in this area is cross-sectional; as such, differences in dependent variables between older and younger individuals that are attributed to age could potentially reflect cohort effects or other confounders. This is a legitimate criticism; however, we believe it is mitigated by the number of studies that have identified similar patterns of results using different samples and age cohorts. We have included longitudinal studies here wherever possible, and suggest that further research in aging and circadian rhythms would benefit greatly from longitudinal designs.

Other considerations pertain to the generalizability of findings made in nocturnal species, such as mice or rats, to diurnal species, such as humans. Although important differences exist in the organization of nocturnal and diurnal circadian systems (16), the high degree of conservation of circadian mechanisms across species supports judicious use of nocturnal animals as a translational model for the human circadian clock (13). Going forward, 


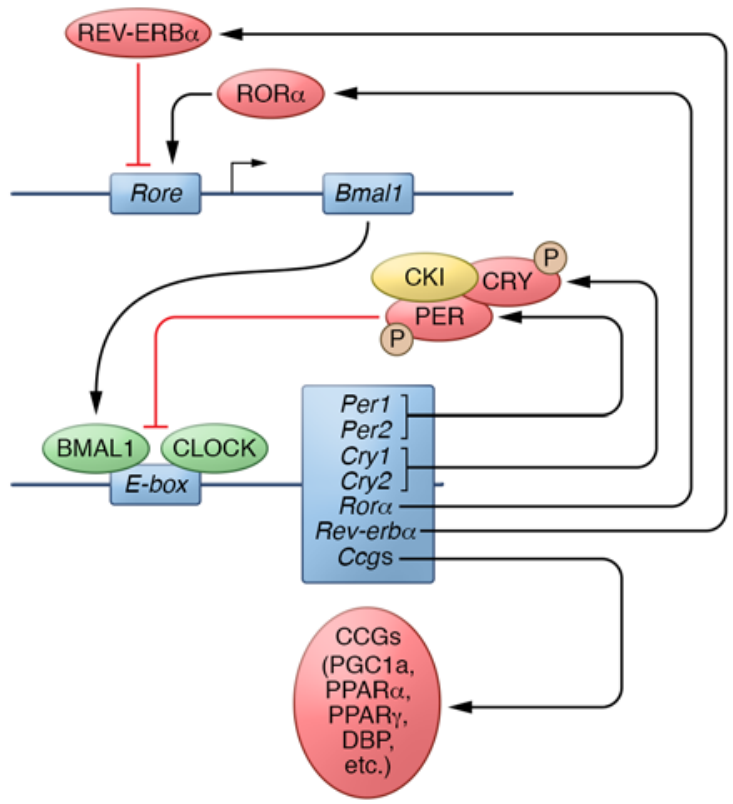

increased use of diurnal species like the grass rat (Arvicanthis ansorgei) in circadian research may enhance the generalizability of findings from animal models to human health.

Activity/rest rhythms. Shifts in preference for morningness versus eveningness, or chronotype, and in sleep cycles are among the most consistently observed age-associated circadian changes in many species. In humans, cross-sectional studies comparing older and younger adults' responses to chronotype assessment tools (17) reliably show that adults in their 60s and above are significantly more likely to endorse a tendency for rising from and retiring to bed earlier than adults in their $20 \mathrm{~s}$ and 30s (18-20). Although there are few longitudinal studies in humans to track individual changes in chronotype with age, those available support the results of cross-sectional designs. Broms and colleagues (21) tracked chronotype longitudinally in 567 adult men in Finland over 23 years (mean age of 56 years at study entry), and found a shift in the distribution toward a "mostly morning" type over years of study. Retrospective self-comparison studies in older adult participants ( $>60$ years) also indicate a tendency to become a "morning person" with increasing age (19). Taken together, this shift in chronotype appears to be a reliable developmental pattern.

The preference for morningness in older adulthood is expressed in other aspects of behavior, such as cognitive skill performance. Performance on recognition memory and reaction time tasks exhibits clear time-of-day changes in accuracy and speed (22). Notably, the circadian profile of cognitive performance

Figure 2. Examples of circadian rhythms in older adults relative to rhythms in younger adults. In the 24-hour cycle, documented changes include rhythms of waking activity; core body temperature; SCN firing; release of hormones, such as melatonin and cortisol; and fasting plasma glucose levels. Relative to younger adults (blue lines), the amplitude of many rhythms dampens in older adults (red lines). In some cases, the peak of the rhythm also advances.

Figure 1. The molecular circadian clock. Heterodimers of the transcription factors BMAL1 and CLOCK upregulate the expression of many target genes. Of these, the protein products of the Period (Per) and Cryptochrome (Cry) genes provide a feedback mechanism to inhibit the transcriptional activity of CLOCK-BMAL1. The activity of PER-CRY dimers is regulated at a posttranscriptional level via phosphorylation by kinases, including casein kinase $1 \varepsilon$ (CKI). Other gene targets of CLOCK-BMAL1 include the nuclear receptors retinoid-related orphan receptor $\alpha(R O R \alpha)$ and REV-ERB $\alpha$, which, respectively, promote and inhibit the transcription of Bmal1. In addition to these core components of the genetic clock, CLOCK-BMAL1 regulates the expression of a number of downstream targets that are referred to as clock-controlled genes (CCGs) $(13,14) \mathrm{P}$, phosphorylation.

interacts with age, such that older adults who are tested on recognition memory tasks in the early morning perform as well as younger adults, but significantly worse when tested later in the afternoon $(23,24)$. This interaction has important implications for the assessment of cognitive functioning in older adults: apparent decrements in cognitive performance may be confounded by testing times in the late afternoon when alertness is decreasing (25).

Impaired phase shifting. The capacity of the circadian clock to accommodate a light/dark schedule change (e.g., rotating shift work or jet lag) varies with age. Studies using a jet lag-type design, in which the timing of light onset between one day and the next is advanced (e.g., a flight from New York to Paris) or delayed by a number of hours, have demonstrated a differential impact of the new schedule on older individuals compared with younger. Following a phase advance, older adults (mean age of 81 years) exhibited decreased sleep efficiency, self-reported alertness, and amplitude of the core body temperature rhythm that persisted for longer compared with younger adults (37-50 years) $(26,27)$. In contrast, a phase delay is better tolerated, with minimal differences observed between older and younger adults (27). Studies in rodents mirror these observations, whereby aged mice (about 2.5 years) subjected to repeated phase advances exhibit poorer glucose tolerance and increased mortality rates compared with younger rodents ( 1 year) or aged rodents subjected to phase delays (28). These age-asso-

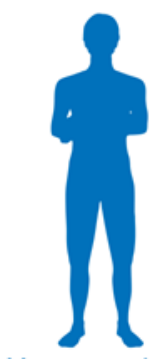

Younger adults

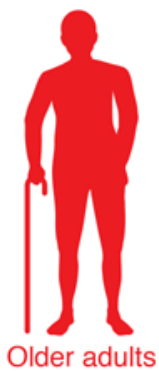

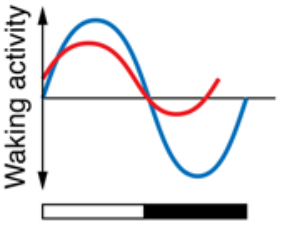
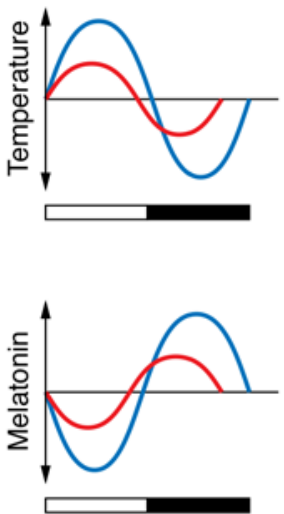
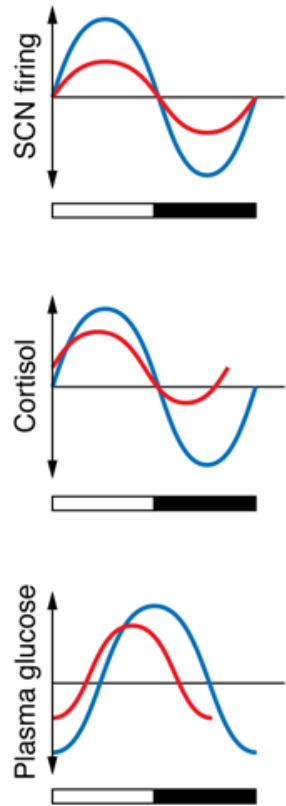


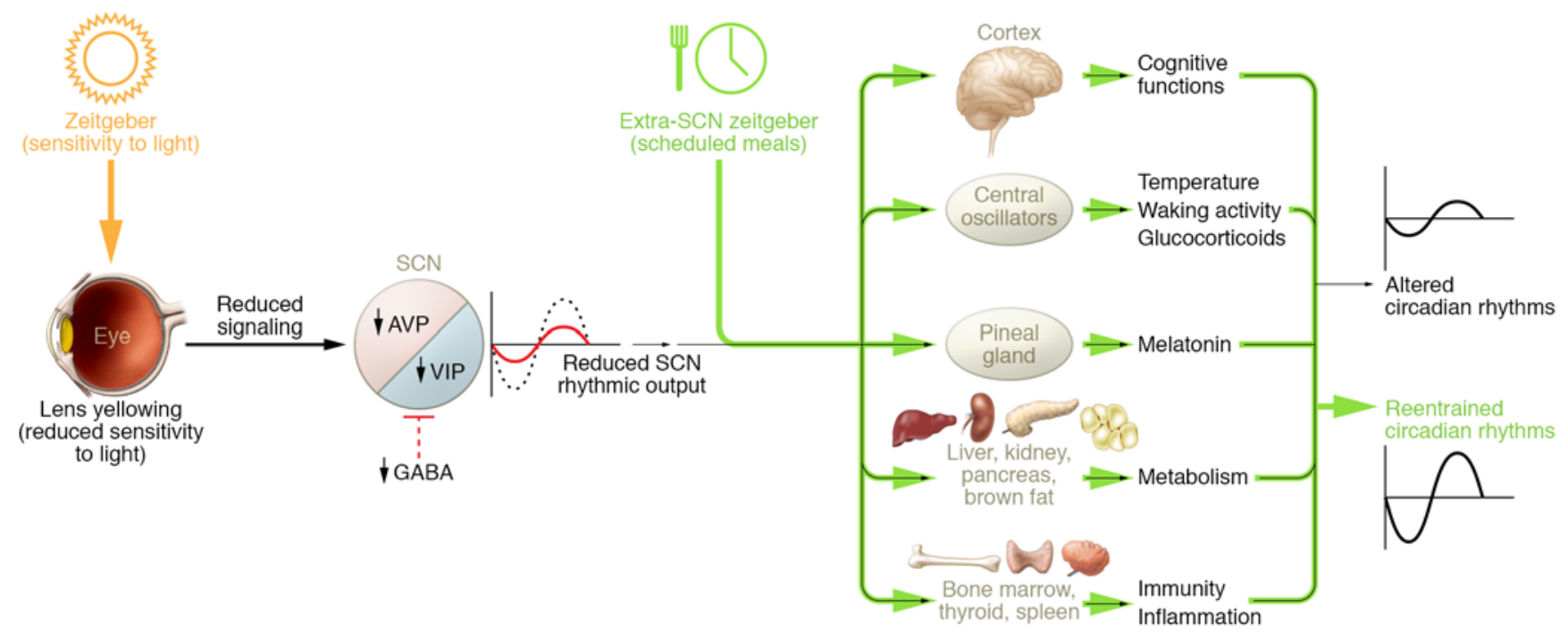

Figure 3. Schematic of possible mechanisms underlying age-related changes in circadian rhythms. Progressive yellowing and thickening of the lens may reduce sensitivity to light, the strongest zeitgeber. Reduction of AVP and VIP expression and fewer CABAergic synapses may decrease signaling within the SCN, leading to a decrease in the overall amplitude of its firing rhythm. A weaker SCN output signal may in turn reduce the strength of downstream oscillators in central and peripheral tissues, including the cortex, pineal gland, liver, kidney, thyroid, and spleen. Providing other zeitgebers such as scheduled meals (green arrows), which act on the circadian system via extra-SCN pathways, may help entrain an aging circadian system.

ciated differences may be attributable to the reduced responsiveness of the aged master circadian clock to light, as illustrated in smaller-magnitude light pulse-induced expression of Per1 mRNA in the SCN of aged hamsters and mice (29-32). Given the prevalence of rotating shift work schedules in industrialized societies, the implications of these findings for healthy aging are significant, and have served as impetus for recent position papers classifying exposure to "light at night" as hazardous to human health (33).

Sleep. Sleep timing and architecture undergo a number of changes in late adulthood. Consistent with the transition to a morning chronotype in older adult humans, the circadian phase of sleep onset and wakening advances with age, whereby older adults (mean age of 68 years) report preferred bedtimes 1 to 2 hours earlier, on average, compared with younger adults (mean age of 23 years) $(34,35)$. Older adults experience significantly more wakenings, have longer latencies to fall asleep, and spend less time in stage 3 , stage 4 , and rapid eye movement (REM) sleep compared with younger adults $(18,36-38)$. Similar observations of sleep fragmentation have been made in aged rhesus monkeys (38), hamsters (39), and Drosophila (40). Van Cauter and colleagues (41) estimate that these age-associated disruptions in human sleep consolidation lead to a loss of approximately 30 minutes of sleep every 10 years, beginning in the fourth decade of life. These changes in amount and quality of sleep likely contribute to an increase in daytime sleepiness experienced by many older adults, which may negatively affect quality of life $(42,43)$ and exacerbate risks of cognitive decline and falls $(44,45)$.

It is important to note that timing of sleep is driven not only by a circadian clock, but also by a homeostatic system that interacts with the circadian rhythm to produce a consolidated period of nightly sleep in humans $(46,47)$. This interaction appears to be highly sensitive to misalignments; as such, age-related changes in this homeostatic mechanism may affect sleep timing, quantity, and quality (48).
Temperature rhythms. In young adult humans (mid-20s), core body temperature rhythm normally peaks in the early evening and reaches its nadir in the early morning (49). In older adults (late $60 \mathrm{~s})$, the period of this rhythm remains stable (50); however, the amplitude of the rhythm decreases in older adult men by $20 \%$ to $40 \%$, such that the nadir of core body temperature does not fall as low as in younger adults $(36,51,52)$ (note that this change has not been observed consistently in older women; ref. 53). In addition to a smaller amplitude, a 1- to 2-hour phase advance in this rhythm has been observed in adults in their 60s to 80s, compared with younger adults in their 20s and 30s $(34,51)$.

Melatonin and cortisol. The circadian profiles of the release of several hormones undergo a number of age-related changes, although supporting evidence is not entirely consistent (54). Of the wide variety of hormones that are under circadian control, melatonin and cortisol are of particular interest, as they have a direct influence in regulating rhythms downstream of the master circadian clock. For example, melatonin release regulates core body temperature, promotes sleep onset, and modulates the activity of intrinsically photosensitive retinal ganglion cells that provide time-keeping signals to the $\operatorname{SCN}(55,56)$. Total melatonin secretion declines with age, beginning perhaps as early as the third decade of life (57, 58). Evidence in humans, rhesus monkeys, and hamsters suggests that the normal nighttime peak in older adults is reduced and shifts to earlier in the evening compared with that in younger adults $(35,38,59,60)$.

However, other evidence suggests that a "young" melatonin rhythm is in fact preserved in very healthy older adults, and a reduction in peak melatonin may occur only in older individuals whose total melatonin levels are at the lower end of the population distribution $(61,62)$. For example, a 6-year longitudinal study found no evidence of a decrease in total melatonin secretion in healthy older adults (ages ranging from 55 to 74 years) (62). This discrepancy in 
findings may suggest that a reduction in the melatonin rhythm is not a feature of a healthy aging process, and in fact may signal underlying pathology. To this end, Waller and colleagues (63) reported a lower nightly melatonin peak in middle-aged men (mean age of 57 years) with cognitive impairment compared with age-matched healthy controls. Furthermore, significant reductions in melatonin synthesis and expression of melatonin receptors in the SCN have been documented in individuals with neurodegenerative diseases such as Alzheimer's or Parkinson's, or those with prodromal symptoms (64-66). The relation between melatonin and age-associated neuropathologies has been explored in recent reviews $(67,68)$.

Like melatonin, rhythmic cortisol release is under control of the SCN (69) and regulates downstream rhythmic expression of clock genes in peripheral tissues in humans, rodents, and dogs (70-74). Cortisol rhythms exhibit age-related changes including a reduction in amplitude (due to higher cortisol secretion at night; refs. 75,76 ), and a phase advance in the peak of cortisol to earlier in the morning (77). Like disruption of the melatonin rhythm, disruption of the cortisol rhythm in aged humans may indicate progressive neurodegeneration (78-80), although not all evidence supports this idea (63).

Metabolism and inflammation. In mammals, circadian clocks within tissues like the liver and pancreas regulate rhythms of metabolism such as glucose homeostasis, lipid metabolism, and xenobiotic detoxification (reviewed in refs. 12, 14). Many metabolic rhythms exhibit progressive dampening with age (76, 81, 82). These changes are underscored by evidence of age-related decreases in the amplitude of clock gene expression rhythms in clocks outside of the master pacemaker $(83,84)$. Dampening of these rhythms is suspected to contribute to the increased risk in older adults of metabolic diseases such as diabetes, dyslipidemia, and hypertension $(12,85)$.

Inflammatory processes, heavily implicated in cellular aging, are also rhythmic. Plasma levels of hematopoietic cells and proinflammatory cytokines are among the components of the immune system that fluctuate in accordance with an animal's rest/activity cycle (reviewed in ref. 86). Recent evidence indicates a direct role for clock genes in regulating inflammation: for example, bone marrow-specific knockout of Bmal1 in mice leads to exaggerated inflammation responses to infection or high-fat diet (87). In response to progressive immunosenescence, chronic, low-grade activation of proinflammatory processes develops within aging organisms, the development of which may increase risk of chronic disease states, such as metabolic syndrome and neurodegenerative diseases (88). Taken together, these findings suggest that age-related changes in circadian clockwork throughout the body may promote chronic inflammation and pathologies including metabolic disorders.

Circadian clock gene expression. In humans and rodents, the expression patterns of clock genes in a variety of tissues exhibit change with age. For example, Chen and colleagues (89) recently reported that in adults over 60 years of age the rhythms of expression of the PERIOD genes PER1 and PER2 in human orbitofrontal cortex are flattened and phase-advanced by approximately 4 to 6 hours, and the expression of CRY1 became arrhythmic, in comparison with adults under 40 years of age. In peripheral blood cells, level of BMAL1 expression was found to correlate negatively with age in women (range 20-79 years) (90).
Findings of age-associated changes in the master clock have been inconsistent in nonhuman species. In Per2-luciferase reporter mice (PER2::LUC mice) housed in constant dark, older (13-15 months) mice demonstrated a rhythm of PER2 protein in the SCN that was of smaller amplitude and shorter period relative to younger mice (3-5 months) (91). These PER2 data conflict with other reports of no differences in SCN rhythms of expression of Period genes Per 1 and Per 2 between young and old animals $(29,84$, 92). However, age-associated reductions were found in the overall expression of Clock and Bmal1 transcripts in the hamster (17-20 months) and mouse (22 months) SCN $(30,93)$.

In contrast, a dampening of rhythmic clock gene activity has been observed in peripheral oscillators in rodents and Drosophila. In tissues such as the lungs, the rhythm of Per1 expression seen in young rats (2 months) was absent in old animals (24-26 months) (84). In Drosophila, the amplitude of clock gene expression and protein rhythms in head and body tissues decreased in aged flies (58 days old), despite the persistence of a robust PER2 rhythm in brain clock neurons (83). These declines in peripheral clocks may have important implications for the susceptibility of aged animals to various disease states, given evidence demonstrating links between loss of rhythmic clock gene expression and the development of metabolic disease states and cancers $(94,95)$.

The interaction of circadian clock genes with the aging process is complex, in that evidence supports a bidirectional relation between them. Not only do clock gene expression patterns appear to change as a function of age, but also these changes may drive the rate of aging. For example, mutations of the clock gene Bmal1 and the Period genes yield an accelerated aging phenotype in Drosophila and mice, with faster rates of tissue decline, impairments in cognitive function, and shorter lifespan relative to age-matched wild-type controls (96-98). The causal role that altered clock gene expression may play in the circadian changes typically observed in aging will be explored further in the next section.

\section{The aging clock: possible mechanisms}

Changes in the SCN. Compelling support for a relation between the master clock and aging comes from transplant experiments, in which introduction of fetal SCN tissue into the brain of an older animal restored dampened rhythms of locomotor activity (99), temperature, drinking, and transcription of corticotropin-releasing hormone in the brain $(100,101)$. Some aged animals receiving fetal tissue grafts exhibit an increased lifespan (99), raising the intriguing possibility that the master clock regulates not only daily rhythms but also longevity.

Morphology and neuropeptide expression. There is mixed evidence in both humans and rodents for age-related changes in the structure of the SCN. Some cross-sectional studies indicated a decrease in SCN volume in adults over 80 years of age compared with younger adults, and fewer total cells in the aged SCN $(102,103)$; however, these findings may have been influenced by the type of cell marker used. In rats, decreased SCN volume and cell number have been reported in some studies (104), but not others (105).

A more consistent body of work indicates age-related changes in the neurochemical makeup of the SCN in humans, primates, and rodents. In particular, the expression of arginine vasopressin 
(AVP) and vasoactive intestinal polypeptide (VIP), each of which follows a circadian rhythm and predominates in the shell and core of the SCN, respectively, is reduced in elderly humans compared with younger and middle-aged adults (refs. 14, 64, 106, 107, and Figure 3). A similar reduction in AVP and VIP expression has been observed in aged rodents $(108,109)$, whereas in mouse lemurs, a nonhuman primate, the peak of rhythmic AVP and VIP expression in the SCN is shifted, not suppressed, in aged animals compared with younger adults (110).

Although the implications of a reduction in AVP and VIP expression are unclear, there are a number of intriguing correlations between neuropeptide expression and the stability of several rhythms. In a sample of elderly humans (mean age of 90 years), VIP expression in the SCN correlated positively with the amplitude of the locomotor activity rhythm (based on actigraphy measures taken within 18 months of death) (111). In aged rats, expression of VIP in the SCN correlated negatively with the amount of time spent in REM sleep (108). These observations provide an interesting parallel to the effects of fetal SCN tissue grafts into aged rodents, whereby a conditional association was observed between the presence of VIP-expressing transplanted cells and successful restoration of locomotor activity, temperature, and drinking rhythms in aged mice (101). Modeling work by Vasalou and colleagues (112) underscores the impact of even modest reductions in the connectivity of VIP neurons in the SCN, in that elimination of only a small amount of long-range VIPergic connections leads to decreased synchrony among cells in the master clock.

At a synaptic level, studies in mice suggest an age-associated reduction in the density of GABAergic synapses in both the core and shell of the SCN (113). Given the role of GABAergic signaling in regulating the activity of SCN cell firing (114), this reduction in synapses could have meaningful implications for age-related changes in the timing of the master clock.

Neuronal coupling in the SCN. Perhaps the most consistent changes observed in the aging SCN are related to the rhythm and synchrony of cell firing. Multiunit recordings in rodents indicate that the amplitude of SCN activity decreases with age, and that this decrease may reflect a loss of coherence in firing patterns across SCN cells in aged animals (115-117). Farajnia and colleagues (118) identified in aged mice (approximately 24 months) greater numbers of neurons within the SCN in which peak firing occurred out of phase with the dominant SCN rhythm in comparison with young mice (aged 3 months). SCN neurons in aged animals were also less inhibited by GABAergic stimulation, which appears consistent with the loss of GABAergic synapses in the SCN with age (113). Observations that the amplitude of the firing rhythm in SCN neurons diminishes with age, while the oscillation of firing in individual SCN neurons between day and night appears to remain fairly robust in aged animals, suggest an age-related decline in the overall coherence of the output signal from the $\operatorname{SCN}(91,119)$. This loss of coherence at a network level within the master clock may contribute to the weakening or desynchronization of oscillators downstream of the SCN.

Clock gene expression in the SCN. Causal links between clock gene expression patterns in the SCN and age-associated changes in circadian rhythms have not been conclusively demonstrated. However, what evidence exists suggests that this relation may be critical. In the PER2::LUC mouse, housing in constant dark conditions revealed a dampening of the PER2 rhythm in the SCN with age (13-15 months), as well as a lengthening of the circadian period of expression (91). Further experiments within this study suggest that these changes in rhythmic PER2 expression in the SCN arise as a consequence of decreased rhythmic cell firing in the SCN (91). Targeted manipulation of clock genes selectively within the SCN yields changes in rhythms of physiology or behavior that approximate some of those observed during the natural course of aging (120). For example, a forebrain-specific deletion of floxed Bmal1 in mice profoundly affected locomotor activity rhythms and peripheral clock synchrony in the absence of a light/dark schedule, despite having no impact on lifespan (121).

Interestingly, system-wide knockouts of clock genes create so-called "accelerated aging" phenotypes in both Drosophila and mice. For example, Bmal1-null mice develop sarcopenia, cataracts, and slowed hair growth, and exhibit a significantly shorter lifespan compared with wild-type age mates (96). Similarly, null mutation of the Per gene in Drosophila is associated with significantly shorter lifespan, as well as greater accumulation of age- and stress-related oxidative damage, compared with wild-type flies (97). Some criticisms of these findings have been raised, in that clock genes such as Bmal1 have important, noncircadian roles in the development of organisms $(122,123)$. As such, a system-wide manipulation of these genes may yield a phenotype that is not exclusively due to disruption of the circadian clock (124). However, these and other associated findings raise the intriguing possibility that changes in clock gene expression may be implicated not only in age-associated changes in circadian rhythms of behavior and physiology, but also more broadly in the processes of aging overall (i.e., irrespective of rhythmicity).

A component of this relation between clock genes and aging in physiology may be via sirtuin 1 (SIRT1), an NAD-dependent protein deacetylase (125) that appears to mediate the effects of caloric restriction on longevity (126). In vitro, SIRT1 regulates the acetylation of Bmal1 and Per 2 in mouse liver $(127,128)$ and human hepatocytes (129). SIRT1 expression in mouse brain and liver decreases with age, and knockout of Sirt1 throughout the brain is associated with reduced Bmal1 and Per 2 mRNA expression in the SCN (125, 129). Furthermore, Sirt1 knockouts display a premature aging phenotype, including disrupted activity rhythms comparable to those in aged (19-22 months) wild-type mice $(125,130)$, as well as shortened lifespan and increased levels of proinflammatory markers in blood (129). In contrast, overexpression of Sirt1 in the mouse brain is associated with elevated Bmall and Per 2 mRNA in the SCN, and a shorter free-run period than in wild-type littermates (125). Taken together, these findings suggest a role for an age-dependent decrease in SIRT1 in mediating changes in the molecular circadian clockwork (125).

\section{Sensitivity to zeitgebers}

Light. Given the importance of time cues, or zeitgebers, in resetting the circadian system on a daily basis, reduced sensitivity to zeitgebers from the environment could contribute to circadian changes in later life. Several avenues of research have examined this possibility with respect to light, the most powerful zeitgeber. 
Humans exert considerable control over their exposure to light in their environment. In general, previous research does not suggest that older adults are exposed to less light during the day relative to younger adults (131), although this may not be the case for adults who are in residential care or nursing facilities (132). Important differences may arise, however, in the transmission of light through younger versus older eyes. For example, the human lens yellows and increases in thickness with age, leading to an estimated $1 \%$ annual reduction in overall light passage to the retina with each year above age 18 (133). This yellowing has a selectively stronger effect on shorter wavelengths of light, which are preferentially absorbed by intrinsically photosensitive retinal ganglion cells (ipRGCs) that transmit time-of-day information to the SCN $(134,135)$, and degree of lens yellowing is associated with reported sleep disturbances in older adults (136). Pupil size and responsiveness of ipRGCs to blue light may diminish with age, although not all findings are consistent $(137,138)$.

Cataracts have also been associated with impaired sleep quality, and removal can exert a beneficial effect on sleep efficiency and the amplitude of the melatonin rhythm in older adults (139-141). However, the magnitude of this effect appears limited and transient, and may be most pronounced in individuals who have particularly poor sleep quality $(140,142)$. Given the sensitivity of the circadian system to shorter-wavelength light (with respect to melatonin release, for example), recent randomized controlled clinical trials have evaluated the circadian effects of "blue-blocking" lenses designed to reduce phototoxicity and risk of light-exacerbated macular degeneration. However, evidence to date has not indicated clear effects of such lenses on measures of sleep efficiency, activity, or melatonin release (143).

Metabolic cues. Although light is the most potent zeitgeber for the circadian system, other cues such as metabolic signals play a powerful role in regulating the circadian clock (144). In contrast to the SCN, circadian oscillators in tissues such as liver, heart, kidney, and lungs will entrain to schedules of restricted food presentation that are out of synchrony with the light/dark schedule (145). Research examining the potency of restricted food delivery as a zeitgeber across the lifespan suggests that responsiveness to this nonphotic time cue may only be modestly affected by age. For example, similarly to young rats, older rats (approximately 24 months) will still develop anticipatory bouts of activity for food presentation on a restricted schedule $(146,147)$.

Given that timed food delivery is a highly potent zeitgeber and does not exert its entrainment effects via the SCN (148), it is possible that timed food delivery could serve as an effective means of resynchronizing circadian timing in older individuals (reviewed in ref. 149). For example, Walcott and Tate (147) reported that low-amplitude, fragmented activity rhythms in aged rats (13-18 months) became more consolidated after a 14-day period of food restriction. Chronic caloric restriction has received significant attention as having "anti-aging" effects in humans and nonhuman species, although protocols are typically focused on limiting overall quantities of food intake instead of their timing per se. Interestingly, recent work in Drosophila indicates that the lifespan-extending effects of chronic dietary restriction are mediated by increased expression of several clock genes in peripheral tissues, which regulate fat metabolism (150).
These and other related findings imply that limited, timed food access may be an avenue through which age-associated pathologies involving the circadian system, such as neurodegenerative diseases, could be mitigated (149).

Desynchronization of downstream oscillators from the master clock. Another possible mechanism underlying circadian disruption with aging pertains to the relative synchrony between SCN and oscillators in central and peripheral tissues. One indication of weakened control by the master clock over peripheral oscillators comes from phase-shifting studies, in which rhythms of sleep, activity, and temperature in older animals, including humans, take longer to re-entrain to a light/dark cycle after a phase advance $(26,130)$. Using the PER2::LUC mouse model, Sellix and colleagues (151) demonstrated that peripheral clocks in esophagus, lung, and thymus gland in older mice (22-28 months) were slower to entrain to a 6-hour phase advance of the light/dark schedule, in comparison with younger (3-6 months) mice. In Drosophila, downstream clock gene oscillation in peripheral tissues and behavioral rhythms decrease in amplitude and become fragmented with increasing age (58 days) (83), despite robust Per expression rhythms persisting in neurons. These findings may suggest that a dampening of rhythmic output from the master clock with age may lead to a desynchronization between central and peripheral oscillators, and consequently misalign peripheral oscillators with external temporal cues (151). In support of this idea, manipulations intended to align peripheral oscillators with the master clock, such as imposing an environmental temperature rhythm that is synchronized with the light/dark cycle, have yielded improvements in sleep and activity rhythms in aged Drosophila (83). Similarly, in rodents lacking a functional master clock, restricted feeding schedules are sufficient to synchronize rhythmic clock gene expression across several peripheral oscillators $(121,152)$.

\section{Conclusions}

The functioning of the biological clock is intimately linked to healthy development across the lifespan. Clearly, a number of rhythms in behavior and physiology exhibit marked flexibility as humans transition from early to late adulthood. Given the centrality of the circadian clock to so many vital human physiological functions, deeper insights into how age-related changes in the clock arise may be far more influential for human health and longevity than anticipated. In support of this, analyses of the variability of the free-run circadian period $(\tau)$ in several species suggest a negative relationship between lifespan and deviation of $\tau$ from 24 hours $(153,154)$.

Going forward, one of the primary challenges will be to discern which among these changes in the circadian system documented to date are clearly maladaptive for healthy aging, and which are amenable to intervention. Sleep rhythms, for example, may present a viable target. Yet whereas sleep disruptions are consistently linked to a number of poorer health outcomes, a shift in chronotype may not be as detrimental to health or quality of life in comparison.

Perhaps greater challenges lie in determining the mechanisms that underlie these changes. In view of the complexity of the circadian clock, it is likely that each age-associated pattern of change reflects an interaction of multiple variables. For example, recent 
evidence for a genetic predisposition toward a morningness chronotype (155) may suggest that the transition toward morningness with increasing age could involve epigenetic mechanisms within the molecular clock, changes in inputs to or outputs of the master clock, downstream oscillator sensitivity to SCN signals, or any combination thereof. As such, future research should consider the interplay of the variety of factors reviewed above, including zeitgeber type, SCN function, and peripheral oscillator sensitivity, in investigating the causes of these changes.

\section{Acknowledgments}

This work was supported by the Natural Sciences and Engineering Research Council of Canada, Les Fonds de la recherche en santé Québec, and the Canadian Institutes for Health Research.

Address correspondence to: Shimon Amir, Concordia University, Richard J. Renaud Science Complex, 7141 Sherbrooke West, Montreal, Quebec H4B 1R6, Canada. Phone: 514.848.2424 ext. 2188; E-mail: shimon.amir@concordia.ca.
1. National Institute on Aging. Global Health and Aging. Report 11-7737. Bethesda, Maryland, USA: NIH, US Departments of Health and Human Services; 2011.

2. Kondratov RV. A role of the circadian system and circadian proteins in aging. Ageing Res Rev. 2007;6(1):12-27.

3. Kondratova AA, Kondratov RV. The circadian clock and pathology of the ageing brain. Nat Rev Neurosci. 2012;13(5):325-335.

4. Mattis J, Sehgal A. Circadian rhythms, sleep, and disorders of aging. Trends Endocrinol Metab. 2016;27(4):192-203.

5. Abbott SM, Videnovic A. Chronic sleep disturbance and neural injury: links to neurodegenerative disease. Nat Sci Sleep. 2016;8:55-61.

6. Stevens RG, Brainard GC, Blask DE, Lockley SW, Motta ME. Breast cancer and circadian disruption from electric lighting in the modern world. CA Cancer J Clin. 2014;64(3):207-218.

7. McFadden E, Jones ME, Schoemaker MJ, Ashworth A, Swerdlow AJ. The relationship between obesity and exposure to light at night: cross-sectional analyses of over 100,000 women in the Breakthrough Generations Study. Am JEpidemiol. 2014;180(3):245-250.

8. Lucassen EA, et al. Environmental 24-hr cycles are essential for health. Curr Biol. 2016;26(14):1843-1853.

9. Morris CJ, Purvis TE, Hu K, Scheer FA. Circadian misalignment increases cardiovascular disease risk factors in humans. Proc Natl Acad Sci U S A. 2016;113(10):E1402-E1411.

10. Arendt J. Biological rhythms during residence in polar regions. Chronobiol Int. 2012;29(4):379-394.

11. Welsh DK, Takahashi JS, Kay SA. Suprachiasmatic nucleus: cell autonomy and network properties. Annu Rev Physiol. 2010;72:551-577.

12. Dibner C, Schibler U. Circadian timing of metabolism in animal models and humans. J Intern Med. 2015;277(5):513-527.

13. Mohawk JA, Green CB, Takahashi JS. Central and peripheral circadian clocks in mammals. Annu Rev Neurosci. 2012;35:445-462.

14. Huang W, Ramsey KM, Marcheva B, Bass J. Circadian rhythms, sleep, and metabolism. J Clin Invest. 2011;121(6):2133-2141.

15. Duffield GE. DNA microarray analyses of circadian timing: the genomic basis of biological time. JNeuroendocrinol. 2003;15(10):991-1002.

16. Challet E. Minireview: Entrainment of the suprachiasmatic clockwork in diurnal and nocturnal mammals. Endocrinology. 2007;148(12):5648-5655.

17. Horne JA, Ostberg O. A self-assessment questionnaire to determine morningness-eveningness in human circadian rhythms. Int J Chronobiol. 1976;4(2):97-110.

18. Carrier J, Monk TH, Buysse DJ, Kupfer DJ. Sleep and morningness-eveningness in the 'middle' years of life (20-59 y). J Sleep Res. 1997;6(4):230-237.

19. Roenneberg T, et al. Epidemiology of the human circadian clock. Sleep Med Rev. 2007;11(6):429-438.

20. Yoon C, May CP, Hasher L. Aging, circadian arousal patterns, and cognition. In: Schwarz N, Park D, Knauper B, Sudman S, eds. Cognition, Aging, and Self-Reports. Philadelphia, Pennsylvania, USA: Psychology Press; 1999:117-143.

21. Broms U, et al. Long-term consistency of diurnal-type preferences among men. Chronobiol Int. 2014;31(2):182-188.

22. Wyatt JK, Ritz-De Cecco A, Czeisler CA, Dijk DJ. Circadian temperature and melatonin rhythms, sleep, and neurobehavioral function in humans living on a 20-h day. Am J Physiol. 1999;277(4 pt 2):R1152-R1163.

23. Schmidt C, Peigneux P, Cajochen C, Collette F. Adapting test timing to the sleep-wake schedule: effects on diurnal neurobehavioral performance changes in young evening and older morning chronotypes. Chronobiol Int. 2012;29(4):482-490.

24. May CP. Synchrony effects in cognition: the costs and a benefit. Psychon Bull Rev. 1999;6(1):142-147.

25. Hasher L, Goldstein D, May CP. It's about time: circadian rhythms, memory, and aging. In: Izawa C, Ohta N, eds. Human Learning and Memory: Advances in Theory and Application: The 4th Tsukuba International Conference on Memory. Mahwah, New Jersey, USA: Lawrence Erlbaum Associates; 2005:199-217.

26. Monk TH, Buysse DJ, Reynolds CF, Kupfer DJ. Inducing jet lag in older people: adjusting to a 6-hour phase advance in routine. Exp Gerontol. 1993;28(2):119-133.

27. Monk TH, Buysse DJ, Carrier J, Kupfer DJ. Inducing jet-lag in older people: directional asymmetry. J Sleep Res. 2000;9(2):101-116.

28. Davidson AJ, Sellix MT, Daniel J, Yamazaki S, Menaker M, Block GD. Chronic jet-lag increases mortality in aged mice. Curr Biol. 2006;16(21):R914-R916.

29. Asai M, et al. Circadian profile of Per gene mRNA expression in the suprachiasmatic nucleus, paraventricular nucleus, and pineal body of aged rats. J Neurosci Res. 2001;66(6):1133-1139.

30. Kolker DE, Fukuyama H, Huang DS, Takahashi JS, Horton TH, Turek FW. Aging alters circadian and light-induced expression of clock genes in golden hamsters. J Biol Rhythms. 2003;18(2):159-169.

31. Zhang Y, Kornhauser JM, Zee PC, Mayo KE, Takahashi JS, Turek FW. Effects of aging on light-induced phase-shifting of circadian behavioral rhythms, fos expression and CREB phosphorylation in the hamster suprachiasmatic nucleus. Neuroscience. 1996;70(4):951-961.

32. Duffy JF, Zeitzer JM, Czeisler CA. Decreased sensitivity to phase-delaying effects of moderate intensity light in older subjects. Neurobiol Aging. 2007;28(5):799-807.

33. IARC Working Group on the Evaluation of Carcinogenic Risks to Humans. Painting, firefighting, and shiftwork. IARC Monogr Eval Carcinog Risks Hum. 2010;98:9-764.

34. Duffy JF, Dijk DJ, Klerman EB, Czeisler CA. Later endogenous circadian temperature nadir relative to an earlier wake time in older people. $\mathrm{Am} \mathrm{J}$ Physiol. 1998;275(5 pt 2):R1478-R1487.

35. Duffy JF, Zeitzer JM, Rimmer DW, Klerman EB, Dijk DJ, Czeisler CA. Peak of circadian melatonin rhythm occurs later within the sleep of older subjects. Am J Physiol Endocrinol Metab. 2002;282(2):E297-E303.

36. Dijk DJ, Duffy JF, Czeisler CA. Contribution of circadian physiology and sleep homeostasis to age-related changes in human sleep. Chronobiol Int. 2000;17(3):285-311.

37. Hayashi Y, Endo S. All-night sleep polygraphic recordings of healthy aged persons: REM and slow-wave sleep. Sleep. 1982;5(3):277-283.

38. Zhdanova IV, Masuda K, Quasarano-Kourkoulis C, Rosene DL, Killiany RJ, Wang S. Aging of intrinsic circadian rhythms and sleep in a diurnal nonhuman primate, Macaca mulatta. J Biol Rhythms. 2011;26(2):149-159.

39. Naylor E, Buxton OM, Bergmann BM, Easton A, Zee PC, Turek FW. Effects of aging on sleep in the golden hamster. Sleep. 1998;21(7):687-693.

40. Koh K, Evans JM, Hendricks JC, Sehgal A. A Drosophila model for age-associated changes in sleep:wake cycles. Proc Natl Acad Sci US A. 2006;103(37):13843-13847.

41. Van Cauter E, Leproult R, Plat L. Age-related changes in slow wave sleep and REM sleep and relationship with growth hormone and cortisol levels in healthy men. JAMA. 2000;284(7):861-868.

42. Carskadon MA, Brown ED, Dement WC. Sleep fragmentation in the elderly: relationship to daytime sleep tendency. Neurobiol Aging. 1982;3(4):321-327.

43. Huang YL, Liu RY, Wang QS, Van Someren EJ, Xu $\mathrm{H}$, Zhou JN. Age-associated difference in circadian sleep-wake and rest-activity rhythms. Physiol Behav. 2002;76(4-5):597-603.

44. Ohayon MM, Vecchierini MF. Daytime sleepiness and cognitive impairment in the elderly population. Arch Intern Med. 2002;162(2):201-208. 
45. Stone KL, et al. Sleep disturbances and risk of falls in older community-dwelling men: the outcomes of Sleep Disorders in Older Men (MrOS Sleep) Study. JAm Geriatr Soc. 2014;62(2):299-305.

46. Borbély AA, Daan S, Wirz-Justice A, Deboer T. The two-process model of sleep regulation: a reappraisal. J Sleep Res. 2016;25(2):131-143.

47. Dijk DJ, Czeisler CA. Contribution of the circadian pacemaker and the sleep homeostat to sleep propensity, sleep structure, electroencephalographic slow waves, and sleep spindle activity in humans. J Neurosci. 1995; 15(5 pt 1):3526-3538.

48. Schmidt C, Peigneux P, Cajochen C. Age-related changes in sleep and circadian rhythms: impact on cognitive performance and underlying neuroanatomical networks. Front Neurol. 2012;3:118.

49. Refinetti R, Menaker M. The circadian rhythm of body temperature. Physiol Behav. 1992;51(3):613-637.

50. Czeisler CA, et al. Stability, precision, and near24-hour period of the human circadian pacemaker. Science. 1999;284(5423):2177-2181.

51. Czeisler CA, et al. Association of sleepwake habits in older people with changes in output of circadian pacemaker. Lancet. 1992;340(8825):933-936.

52. Vitiello MV, Smallwood RG, Avery DH, Pascualy RA, Martin DC, Prinz PN. Circadian temperature rhythms in young adult and aged men. Neurobiol Aging. 1986;7(2):97-100.

53. Monk TH, Buysse DJ, Reynolds CF 3rd, Kupfer DJ, Houck PR. Circadian temperature rhythms of older people. Exp Gerontol. 1995;30(5):455-474.

54. Touitou Y, Haus E. Alterations with aging of the endocrine and neuroendocrine circadian system in humans. Chronobiol Int. 2000;17(3):369-390.

55 . Arendt J. Melatonin and human rhythms. Chronobiol Int. 2006;23(1-2):21-37.

56. Pack W, Hill DD, Wong KY. Melatonin modulates M4-type ganglion-cell photoreceptors. Neuroscience. 2015;303:178-188.

57. Kennaway DJ, Lushington K, Dawson D, Lack L, van den Heuvel C, Rogers N. Urinary 6-sulfatoxymelatonin excretion and aging: new results and a critical review of the literature. J Pineal Res. 1999;27(4):210-220.

58. Zhao ZY, Xie Y, Fu YR, Bogdan A, Touitou Y. Aging and the circadian rhythm of melatonin: a cross-sectional study of Chinese subjects 30-110 yr of age. Chronobiol Int. 2002;19(6):1171-1182.

59. Touitou Y, et al. Age- and mental health-related circadian rhythms of plasma levels of melatonin, prolactin, luteinizing hormone and follicle-stimulating hormone in man. JEndocrinol. 1981;91(3):467-475.

60. Reiter RJ, Richardson BA, Johnson LY, Ferguson BN, Dinh DT. Pineal melatonin rhythm: reduction in aging Syrian hamsters. Science. 1980;210(4476):1372-1373.

61. Zeitzer JM, Daniels JE, Duffy JF, et al. Do plasma melatonin concentrations decline with age? Am J Med. 1999;107(5):432-436.

62. Kin NM, Nair NP, Schwartz G, Thavundayil JX,
Annable L. Secretion of melatonin in healthy elderly subjects: a longitudinal study. Ann NY Acad Sci. 2004;1019:326-329.

63. Waller KL, et al. Melatonin and cortisol profiles in late midlife and their association with age-related changes in cognition. Nat Sci Sleep. 2016;8:47-53.

64. Wu YH, Zhou JN, Van Heerikhuize J, Jockers R, Swaab DF. Decreased MT1 melatonin receptor expression in the suprachiasmatic nucleus in aging and Alzheimer's disease. Neurobiol Aging 2007;28(8):1239-1247.

65. Videnovic A, et al. Circadian melatonin rhythm and excessive daytime sleepiness in Parkinson disease. JAMA Neurol. 2014;71(4):463-469.

66. $\mathrm{Wu} \mathrm{YH}$, et al. Molecular changes underlying reduced pineal melatonin levels in Alzheimer disease: alterations in preclinical and clinical stages. J Clin Endocrinol Metab. 2003;88(12):5898-5906

67. Videnovic A, Lazar AS, Barker RA, Overeem S. 'The clocks that time us' - circadian rhythms in neurodegenerative disorders. Nat Rev Neurol. 2014;10(12):683-693.

68. Videnovic A, Zee PC. Consequences of Circadian Disruption on Neurologic Health. Sleep Med Clin. 2015;10(4):469-480.

69 . Oster $\mathrm{H}$, et al. The circadian rhythm of glucocorticoids is regulated by a gating mechanism residing in the adrenal cortical clock. Cell Metab. 2006;4(2):163-173.

70. Cuesta M, Cermakian N, Boivin DB. Glucocorticoids entrain molecular clock components in human peripheral cells. FASEB J. 2015;29(4):1360-1370.

71. Amir S, Lamont EW, Robinson B, Stewart J. A circadian rhythm in the expression of PERIOD2 protein reveals a novel SCN-controlled oscillator in the oval nucleus of the bed nucleus of the stria terminalis. J Neurosci. 2004;24(4):781-790.

72. Segall LA, Perrin JS, Walker CD, Stewart J, Amir S. Glucocorticoid rhythms control the rhythm of expression of the clock protein, Period2, in oval nucleus of the bed nucleus of the stria terminalis and central nucleus of the amygdala in rats. Neuroscience. 2006;140(3):753-757.

73. Balsalobre A, et al. Resetting of circadian time in peripheral tissues by glucocorticoid signaling. Science. 2000;289(5488):2344-2347.

74. Ohmori K, et al. Circadian rhythms and the effect of glucocorticoids on expression of the clock gene period 1 in canine peripheral blood mononuclear cells. Vet J. 2013;196(3):402-407.

75. Touitou Y, et al. Adrenal circadian system in young and elderly human subjects: a comparative study. JEndocrinol. 1982;93(2):201-210.

76. Van Cauter E, Leproult R, Kupfer DJ. Effects of gender and age on the levels and circadian rhythmicity of plasma cortisol. J Clin Endocrinol Metab. 1996;81(7):2468-2473.

77. Sherman B, Wysham C, Pfohl B. Age-related changes in the circadian rhythm of plasma cortisol in man. J Clin Endocrinol Metab. 1985;61(3):439-443

78. Breen DP, et al. Sleep and circadian rhythm regulation in early Parkinson disease. JAMA Neurol. 2014;71(5):589-595.
79. Hartmann A, Veldhuis JD, Deuschle M, Standhardt H, Heuser I. Twenty-four hour cortisol release profiles in patients with Alzheimer's and Parkinson's disease compared to normal controls: ultradian secretory pulsatility and diurnal variation. Neurobiol Aging. 1997;18(3):285-289.

80. Hatfield CF, Herbert J, van Someren EJ, Hodges JR, Hastings MH. Disrupted daily activity/rest cycles in relation to daily cortisol rhythms of home-dwelling patients with early Alzheimer's dementia. Brain. 2004;127(Pt 5):1061-1074.

81. Wijsman CA, et al. Ambulant 24-h glucose rhythms mark calendar and biological age in apparently healthy individuals. Aging Cell. 2013;12(2):207-213

82. Singh R, Sharma S, Singh RK, Cornelissen G. Circadian time structure of circulating plasma lipid components in healthy indians of different age groups. Indian J Clin Biochem. 2016;31(2):215-223.

83. Luo W, et al. Old flies have a robust central oscillator but weaker behavioral rhythms that can be improved by genetic and environmental manipulations. Aging Cell. 2012;11(3):428-438.

84. Yamazaki S, Straume M, Tei H, Sakaki Y, Menaker M, Block GD. Effects of aging on central and peripheral mammalian clocks. Proc Natl Acad Sci U S A. 2002;99(16):10801-10806.

85. Sohail S, Yu L, Bennett DA, Buchman AS, Lim AS. Irregular 24-hour activity rhythms and the metabolic syndrome in older adults. Chronobiol Int. 2015;32(6):802-813.

86. Scheiermann C, Kunisaki Y, Frenette PS. Circadian control of the immune system. Nat Rev Immunol. 2013;13(3):190-198.

87. Nguyen KD, Fentress SJ, Qiu Y, Yun K, Cox JS, Chawla A. Circadian gene Bmal1 regulates diurnal oscillations of Ly6C(hi) inflammatory monocytes. Science. 2013;341(6153):1483-1488.

88. Deleidi M, Jäggle M, Rubino G. Immune aging, dysmetabolism, and inflammation in neurological diseases. Front Neurosci. 2015;9:172.

89. Chen CY, et al. Effects of aging on circadian patterns of gene expression in the human prefrontal cortex. Proc Natl Acad Sci US A. 2016;113(1):206-211.

90. Ando H, et al. Influence of age on clock gene expression in peripheral blood cells of healthy women. J Gerontol A Biol Sci Med Sci. 2010;65(1):9-13.

91. Nakamura TJ, et al. Age-related changes in the circadian system unmasked by constant conditions(1,2,3). eNeuro. 2015;2(4):(4).

92. Kolker DE, Vitaterna MH, Fruechte EM, Takahashi JS, Turek FW. Effects of age on circadian rhythms are similar in wild-type and heterozygous Clock mutant mice. Neurobiol Aging. 2004;25(4):517-523.

93. Bonaconsa M, Malpeli G, Montaruli A, Carandente F, Grassi-Zucconi G, Bentivoglio M. Differential modulation of clock gene expression in the suprachiasmatic nucleus, liver and heart of aged mice. Exp Gerontol. 2014;55:70-79.

94. Marcheva B, et al. Disruption of the clock components CLOCK and BMAL1 leads to hypoinsulinaemia and diabetes. Nature. 2010;466(7306):627-631. 
95. Papagiannakopoulos T, et al. Circadian rhythm disruption promotes lung tumorigenesis. Cell Metab. 2016;24(2):324-331.

96. Kondratov RV, Kondratova AA, Gorbacheva VY, Vykhovanets OV, Antoch MP. Early aging and age-related pathologies in mice deficient in BMAL1, the core componentof the circadian clock. Genes Dev. 2006;20(14):1868-1873.

97. Krishnan N, Kretzschmar D, Rakshit K, Chow E, Giebultowicz JM. The circadian clock gene period extends healthspan in aging Drosophila melanogaster. Aging (Albany NY). 2009;1(11):937-948.

98. Nakahata Y, Sahar S, Astarita G, Kaluzova M, Sassone-Corsi P. Circadian control of the $\mathrm{NAD}^{+}$ salvage pathway by CLOCK-SIRT1. Science. 2009;324(5927):654-657.

99. Hurd MW, Ralph MR. The significance of circadian organization for longevity in the golden hamster. J Biol Rhythms. 1998;13(5):430-436.

100.Cai A, Scarbrough K, Hinkle DA, Wise PM. Fetal grafts containing suprachiasmatic nuclei restore the diurnal rhythm of CRH and POMC mRNA in aging rats. Am J Physiol. 1997;273(5 pt 2):R1764-R1770.

101.Li H, Satinoff E. Fetal tissue containing the suprachiasmatic nucleus restores multiple circadian rhythms in old rats. Am J Physiol. 1998;275(6 pt 2):R1735-R1744.

102. Swaab DF, Fliers E, Partiman TS. The suprachiasmatic nucleus of the human brain in relation to sex, age and senile dementia. Brain Res. 1985;342(1):37-44.

103. Zhou JN, Swaab DF. Activation and degeneration during aging: a morphometric study of the human hypothalamus. Microsc Res Tech. 1999;44(1):36-48.

104. Tsukahara S, Tanaka S, Ishida K, Hoshi N, Kitagawa H. Age-related change and its sex differences in histoarchitecture of the hypothalamic suprachiasmatic nucleus of F344/N rats. Exp Gerontol. 2005;40(3):147-155.

105. Madeira MD, Sousa N, Santer RM, Paula-Barbosa MM, Gundersen HJ. Age and sex do not affect the volume, cell numbers, or cell size of the suprachiasmatic nucleus of the rat: an unbiased stereological study. J Comp Neurol. 1995;361(4):585-601.

106. Hofman MA, Swaab DF. Alterations in circadian rhythmicity of the vasopressin-producing neurons of the human suprachiasmatic nucleus (SCN) with aging. Brain Res. 1994;651(1-2):134-142.

107. Zhou JN, Hofman MA, Swaab DF. VIP neurons in the human SCN in relation to sex, age, and Alzheimer's disease. Neurobiol Aging. 1995;16(4):571-576.

108. Chee CA, Roozendaal B, Swaab DF, Goudsmit E, Mirmiran M. Vasoactive intestinal polypeptide neuron changes in the senile rat suprachiasmatic nucleus. Neurobiol Aging. 1988;9(3):307-312.

109. Roozendaal B, van Gool WA, Swaab DF, Hoogendijk JE, Mirmiran M. Changes in vasopressin cells of the rat suprachiasmatic nucleus with aging. Brain Res. 1987;409(2):259-264.

110. Cayetanot F, Bentivoglio M, Aujard F. Arginine-vasopressin and vasointestinal polypeptide rhythms in the suprachiasmatic nucleus of the mouse lemur reveal aging-related alterations of circadian pacemaker neurons in a non-human primate. Eur J Neurosci. 2005;22(4):902-910.

111. Wang JL, et al. Suprachiasmatic neuron numbers and rest-activity circadian rhythms in older humans. Ann Neurol. 2015;78(2):317-322.

112. Vasalou C, Herzog ED, Henson MA. Smallworld network models of intercellular coupling predict enhanced synchronization in the suprachiasmatic nucleus. J Biol Rhythms. 2009;24(3):243-254

113. Palomba $M$, Nygård $M$, Florenzano $F$, Bertin G, Kristensson K, Bentivoglio M. Decline of the presynaptic network, including GABAergic terminals, in the aging suprachiasmatic nucleus of the mouse. J Biol Rhythms. 2008;23(3):220-231.

114. Aton SJ, Huettner JE, Straume M, Herzog ED. GABA and Gi/o differentially control circadian rhythms and synchrony in clock neurons. Proc Natl Acad Sci U S A. 2006;103(50):19188-19193.

115. Satinoff E, et al. Do the suprachiasmatic nuclei oscillate in old rats as they do in young ones? Am J Physiol. 1993;265(5 pt 2):R1216-R1222.

116. Watanabe A, Shibata S, Watanabe S. Circadian rhythm of spontaneous neuronal activity in the suprachiasmatic nucleus of old hamster in vitro. Brain Res. 1995;695(2):237-239.

117. Nakamura TJ, et al. Age-related decline in circadian output. J Neurosci. 2011;31(28):10201-10205.

118. Farajnia S, et al. Evidence for neuronal desynchrony in the aged suprachiasmatic nucleus clock. J Neurosci. 2012;32(17):5891-5899.

119. Nakamura TJ, Takasu NN, Nakamura W. The suprachiasmatic nucleus: age-related decline in biological rhythms. J Physiol Sci. 2016;66(5):367-374.

120. Gavrila AM, Robinson B, Hoy J, Stewart J, Bhargava A, Amir S. Double-stranded RNA-mediated suppression of Period 2 expression in the suprachiasmatic nucleus disrupts circadian locomotor activity in rats. Neuroscience. 2008;154(2):409-414.

121. Izumo M, et al. Differential effects of light and feeding on circadian organization of peripheral clocks in a forebrain Bmal1 mutant. Elife. 2014;3.

122. Banks G, Nolan PM, Peirson SN. Reciprocal interactions between circadian clocks and aging. Mamm Genome. 2016;27(7-8):332-340.

123. McDearmon EL, et al. Dissecting the functions of the mammalian clock protein BMAL1 by tissue-specific rescue in mice. Science. 2006;314(5803):1304-1308.

124. Yang G, et al. Timing of expression of the core clock gene Bmal1 influences its effects on aging and survival. Sci Transl Med. 2016;8(324):324ra16.

125. Chang HC, Guarente L. SIRT1 mediates central circadian control in the SCN by a mechanism that decays with aging. Cell. 2013;153(7):1448-1460.

126. Finkel T, Deng CX, Mostoslavsky R. Recent progress in the biology and physiology of sirtuins. Nature. 2009;460(7255):587-591.

127. Nakahata $\mathrm{Y}$, et al. The NAD ${ }^{+}$-dependent deacetylase SIRT1 modulates CLOCK-mediated chromatin remodeling and circadian control. Cell. 2008;134(2):329-340.

128. Asher G, et al. SIRT1 regulates circadian clock gene expression through PER2 deacetylation.
Cell. 2008;134(2):317-328.

129.Wang RH, et al. Negative reciprocal regulation between Sirt1 and Per2 modulates the circadian clock and aging. Sci Rep. 2016;6:28633.

130. Valentinuzzi VS, Scarbrough K, Takahashi JS, Turek FW. Effects of aging on the circadian rhythm of wheel-running activity in C57BL/6 mice. Am J Physiol.1997;273(6 pt 2):R1957-R1964.

131. Scheuermaier K, Laffan AM, Duffy JF. Light exposure patterns in healthy older and young adults. J Biol Rhythms. 2010;25(2):113-122.

132. Shochat T, Martin J, Marler M, Ancoli-Israel S. Illumination levels in nursing home patients: effects on sleep and activity rhythms. J Sleep Res. 2000;9(4):373-379.

133. Kessel L, Lundeman JH, Herbst K, Andersen TV, Larsen M. Age-related changes in the transmission properties of the human lens and their relevance to circadian entrainment. J Cataract Refract Surg. 2010;36(2):308-312.

134. Hattar S, et al. Melanopsin and rod-cone photoreceptive systems account for all major accessory visual functions in mice. Nature. 2003;424(6944):76-81.

135. Hattar S, Liao HW, Takao M, Berson DM, Yau KW Melanopsin-containing retinal ganglion cells: architecture, projections, and intrinsic photosensitivity. Science. 2002;295(5557):1065-1070.

136. Kessel L, Siganos G, Jørgensen T, Larsen M. Sleep disturbances are related to decreased transmission of blue light to the retina caused by lens yellowing. Sleep. 2011;34(9):1215-1219.

137. Herbst K, et al. Intrinsically photosensitive retinal ganglion cell function in relation to age: a pupillometric study in humans with special reference to the age-related optic properties of the lens. BMC Ophthalmol. 2012;12:4.

138. Kankipati L, Girkin CA, Gamlin PD. Post-illumination pupil response in subjects without ocular disease. Invest Ophthalmol Vis Sci. 2010;51(5):2764-2769.

139. Asplund R, Lindblad BE. Sleep and sleepiness 1 and 9 months after cataract surgery. Arch Gerontol Geriatr. 2004;38(1):69-75.

140.Ayaki M, Muramatsu M, Negishi K, Tsubota $\mathrm{K}$. Improvements in sleep quality and gait speed after cataract surgery. Rejuvenation Res. 2013;16(1):35-42.

141. Brøndsted AE, Lundeman JH, Kessel L. Short wavelength light filtering by the natural human lens and IOLs - implications for entrainment of circadian rhythm. Acta Ophthalmol. 2013;91(1):52-57.

142. Brøndsted AE, et al. The effect of cataract surgery on circadian photoentrainment: a randomized trial of blue-blocking versus neutral intraocular lenses. Ophthalmology. 2015;122(10):2115-2124.

143. Yan SS, Wang W. The effect of lens aging and cataract surgery on circadian rhythm. Int JOphthalmol. 2016;9(7):1066-1074.

144. Stephan FK. The "other" circadian system: food as a Zeitgeber. JBiol Rhythms. 2002;17(4):284-292.

145. Damiola F, Le Minh N, Preitner N, Kornmann B, Fleury-Olela F, Schibler U. Restricted feeding uncouples circadian oscillators in peripheral tissues from the central pacemaker in the suprachiasmatic nucleus. Genes Dev. 
2000;14(23):2950-2961.

146. Mistlberger RE, Houpt TA, Moore-Ede MC. Effects of aging on food-entrained circadian rhythms in the rat. Neurobiol Aging. 1990;11(6):619-624.

147. Walcott EC, Tate BA. Entrainment of aged, dysrhythmic rats to a restricted feeding schedule. Physiol Behav. 1996;60(5):1205-1208.

148. Boulos Z, Rosenwasser AM, Terman M. Feeding schedules and the circadian organization of behavior in the rat. Behav Brain Res. 1980;1(1):39-65.

149. Kent BA. Synchronizing an aging brain: can entraining circadian clocks by food slow Alzheimer's disease? Front Aging Neurosci. 2014;6:234.

150. Katewa SD, et al. Peripheral circadian clocks mediate dietary restriction-dependent changes in lifespan and fat metabolism in Drosophila. Cell Metab. 2016;23(1):143-154.

151. Sellix MT, et al. Aging differentially affects the re-entrainment response of central and peripheral circadian oscillators. J Neurosci. 2012;32(46):16193-16202.

152. Tahara $\mathrm{Y}$, et al. In vivo monitoring of peripheral circadian clocks in the mouse. Curr Biol. 2012;22(11):1029-1034
153. Wyse CA, Coogan AN, Selman C, Hazlerigg DG, Speakman JR. Association between mammalian lifespan and circadian free-running period: the circadian resonance hypothesis revisited. Biol Lett. 2010;6(5):696-698.

154. Libert S, Bonkowski MS, Pointer K, Pletcher SD, Guarente L. Deviation of innate circadian period from $24 \mathrm{~h}$ reduces longevity in mice. Aging Cell. 2012;11(5):794-800.

155. Jones SE, et al. Genome-wide association analyses in 128,266 individuals identifies new morningness and sleep duration loci. PLoS Genet. 2016;12(8):e1006125 